\title{
On Lifted Cover Inequalities: A New Lifting Procedure with Unusual Properties
}

\author{
Adam N. Letchford* Georgia Souli ${ }^{\dagger}$ \\ To appear in Operations Research Letters
}

\begin{abstract}
Lifted cover inequalities are well-known cutting planes for 0-1 linear programs. We show how one of the earliest lifting procedures, due to Balas, can be significantly improved. The resulting procedure has some unusual properties. For example, (i) it can yield facet-defining inequalities even if the given cover is not minimal, (ii) it can yield facet-defining inequalities that cannot be obtained by standard lifting procedures, and (iii) the associated superadditive lifting function is integer-valued almost everywhere.
\end{abstract}

Keywords: knapsack problems; lifted cover inequalities; polyhedral combinatorics

\section{Introduction}

Strong valid linear inequalities, also called cutting planes, are a key ingredient of modern exact algorithms for integer programs (see, e.g., [5, 4]). In the case of pure 0-1 linear programs (0-1 LPs), one very well-known and widely used family of cutting planes is the so-called lifted cover inequalities (LCIs), discovered independently by Balas [2] and Wolsey [16] (see also the surveys [1, 14]).

LCIs are obtained from a weaker family of inequalities, the so-called cover inequalities (CIs), by a process called lifting. Several procedures for lifting CIs have been proposed in the literature [2, 3, 7, 9, 10, 13, 18. In this paper, we focus on one of the earliest lifting procedures, which was described in Section 3 of Balas 2. The LCIs generated by Balas' procedure are not guaranteed to define facets of the associated knapsack polytope, but they tend to be strong in practice. Moreover, the procedure is extremely

\footnotetext{
${ }^{*}$ Corresponding author. Department of Management Science, Lancaster University, Lancaster LA1 4YX, United Kingdom. E-mail: a.n.letchford@lancaster.ac.uk

${ }^{\dagger}$ STOR-i Centre for Doctoral Training, Lancaster University, Lancaster LA1 4YR, United Kingdom. E-mail: G.Souli@lancaster.ac.uk
} 
fast. Specifically, it runs in only $O(n \log c)$ time, where $n$ is the number of variables and $c$ is the number of items in the cover.

The purpose of this paper is to show that the above-mentioned lifting procedure of Balas can be significantly improved, so that it yields both stronger and more general LCIs, while still running in only $O(n \log c)$ time. The improved procedure is sequence-independent, and it has some unusual properties:

- It can increase coefficients for variables inside the cover as well as outside.

- It can yield facet-defining LCIs even if the given cover is not minimal.

- It can even yield facet-defining inequalities that cannot be obtained by standard lifting procedures.

- The associated lifting function is integer-valued almost everywhere.

Moreover, the proof that the improved procedure is valid is itself unusual. It relies on the use of "dummy variables", by which we mean variables that do not exist in reality.

The paper is structured as follows. In Section 2, we review the literature. In Section 3, we present and analyse the improved lifting procedure. In Section 4 , we show how to further enhance the procedure, via an analysis of superadditive functions.

Throughout the paper, $x_{1}, \ldots, x_{n}$ will be a collection of binary variables and $N$ will denote $\{1, \ldots, n\}$. Moreover, given a vector $v \in \mathbb{Q}_{+}^{n}$ and a set $S \subseteq N$, we will let $v(S)$ denote $\sum_{j \in S} v_{j}$.

\section{Literature Review}

We now briefly review the relevant literature.

\subsection{Lifted cover inequalities}

A knapsack constraint is a linear constraint of the form $\sum_{j \in N} a_{j} x_{j} \leq b$, where $a \in \mathbb{Z}_{+}^{n}$ and $b$ is a positive integer. Any linear inequality involving binary variables can be converted into a knapsack constraint, by complementing variables with negative coefficients [16]. The polyhedron

$$
\operatorname{conv}\left\{x \in\{0,1\}^{n}: \sum_{j \in N} a_{j} x_{j} \leq b\right\}
$$

is called a knapsack polytope [2].

A set $C \subseteq N$ such that $a(C)>b$ is called a cover. If $C$ is a cover, then the inequality $x(C) \leq|C|-1$ is valid for the knapsack polytope [8]. It is 
called a cover inequality (CI). A cover (and the associated CI) is minimal if $a(C \backslash\{k\}) \leq b$ for all $k \in C$. Minimal CIs dominate all other CIs.

Unfortunately, minimal CIs do not in general define facets of the knapsack polytope. On the other hand, given any minimal cover $C$, there exists at least one facet-defining lifted cover inequality (LCI) of the form

$$
x(C)+\sum_{j \in N \backslash C} \alpha_{j} x_{j} \leq|C|-1,
$$

where $\alpha_{j} \in \mathbb{Z}_{+}$for $j \in N \backslash C$ [2, 15, 16]. (There may also exist facet-defining LCIs in which some $\alpha_{j}$ are fractional.) The process of computing the $\alpha_{j}$ for $j \in N \backslash C$ is called lifting. Encouraging computational results with LCIs were given in [7].

One can define more general LCIs of the form

$$
x(C \backslash D)+\sum_{j \in N \backslash C} \alpha_{j} x_{j}+\sum_{j \in D} \beta_{j} x_{j} \leq|C \backslash D|-1+\beta(D),
$$

where $D \subset C[15,16]$. Encouraging computational results with general LCIs are given in [9, 12, 13]. We will follow Gu et al. 9] in calling the computation of the $\alpha_{j}$ and $\beta_{j}$ up-lifting and down-lifting, respectively. We will also call LCIs of the form (1) simple.

\subsection{Balas' lifting procedure}

Balas [2] introduced the following elegant up-lifting procedure, which can be implemented to run in $O(n \log |C|)$ time. Let $C$ be a minimal cover and, for $r=1, \ldots,|C|$, let $S(r)$ be the sum of the $r$ largest $a_{j}$ values over the members of $C$. Also let $S(0)=0$. Given any $j \in N \backslash C$, let $\lambda_{j}$ be the (unique) integer such that $S\left(\lambda_{j}\right) \leq a_{j}<S\left(\lambda_{j}+1\right)$. Then the simple LCI

$$
x(C)+\sum_{j \in N \backslash C} \lambda_{j} x_{j} \leq|C|-1
$$

is valid.

Balas \& Zemel [3] proved the following stronger result. Given any $j \in$ $N \backslash C$, let $\mu_{j}$ be the (unique) integer such that

$$
a(C)-S\left(\mu_{k}+1\right) \leq b-a_{k}<a(C)-S\left(\mu_{k}\right) .
$$

Then, in any facet-defining simple LCI, we have $\lambda_{j} \leq \alpha_{j} \leq \mu_{j} \leq \lambda_{j}+1$ for all $j \in N \backslash C$.

We will use the following example at several points through the paper.

Example 1. Let $n=10, a=(15,13,9,8,8,8,5,5,5,5)$ and $b=16$. The set $\{7,8,9,10\}$ is a minimal cover. We have $S(k)=5 k$ for $k=0, \ldots, 4$. Since 
$a_{1}=15 \geq S(3)$, we have $\lambda_{1}=3$. Since $a_{2}=13 \geq S(2)$, we have $\lambda_{2}=2$. On the other hand, since $a_{3}, \ldots, a_{6}<S(2)$, we have $\lambda_{3}, \ldots, \lambda_{6}=1$. The resulting (simple) LCI is therefore

$$
3 x_{1}+2 x_{2}+x_{3}+\cdots+x_{10} \leq 3 .
$$

One can also check that $\mu_{1}=\mu_{2}=3$ and $\mu_{k}=2$ for $k \in\{3,4,5,6\}$. So, in any facet-defining LCI obtained from that specific cover, $\alpha_{1}=3, \alpha_{2} \in[2,3]$ and $\alpha_{3}, \alpha_{4}, \alpha_{5}, \alpha_{6} \in[1,2]$.

\subsection{Other lifting procedures}

It was shown in [15, 16] that one can obtain at least one facet-defining simple LCI by performing up-lifting sequentially, i.e., one coefficient at a time. This can be done by solving a small knapsack problem for each variable in $N \backslash C$. Zemel [18] showed how to do it in $O(n|C|)$ time by dynamic programming.

It is also possible to obtain facet-defining simple LCIs with fractional coefficients, by up-lifting simultaneously instead of sequentially. Unfortunately, this is not easy. In fact, even recognising a facet-defining simple LCI obtained by simultaneous up-lifting is $\mathcal{N} \mathcal{P}$-hard [1].

It is of course possible to perform simultaneous up-lifting approximately in polynomial time. If an approximate simultaneous up-lifting procedure does not require any ordering of the variables in $N \backslash C$, it is called sequenceindependent [3, 10]. The procedure of Balas, described in the previous subsection, can be viewed as a simple sequence-independent up-lifting procedure.

Wolsey [17] established a connection between sequence-independent lifting and superadditive functions. Gu et al. [10] used that result to improve Balas' up-lifting procedure, without increasing the asymptotic running time. Their improved procedure can yield simple LCIs with fractional coefficients. Further results on lifting can be found in [9, 12, 14].

\section{The New Procedure and Its Properties}

In this section, we show how to improve the procedure of Balas [2], in a way that is different from the one given in [10]. Throughout this section, we assume that we have a fixed knapsack constraint $a^{T} x \leq b$ and a fixed (not necessarily minimal) cover $C$, for ease of notation. We let $c$ and $a_{\max }$ denote $|C|$ and $\max _{j \in C}\left\{a_{j}\right\}$, respectively. Finally, we assume that the items in $C$ have been sorted in non-increasing order of $a_{j}$ value, and we let $\ell_{1}, \ldots, \ell_{c}$ be the sorted values. Note that the sorting can be performed in $O(c \log c)$ time. 


\subsection{A key quantity}

The following quantity will play a crucial role in our analysis.

Definition 1 We let $\bar{a}$ denote the unique (positive and rational) number such that

$$
\sum_{j \in C} \min \left\{a_{j}, \bar{a}\right\}=b
$$

For example, if $C=\{1,3,4\}, a_{1}=10, a_{3}=7, a_{4}=5$ and $b=18$, then $\bar{a}=6.5$, since $6.5+6.5+5=18$.

Remark 1 We have $\frac{b}{c} \leq \bar{a}<a_{\max }$.

Remark 2 If the items in $C$ have already been sorted, one can compute $\bar{a}$ in $O(c)$ time. See Algorithm 1 .

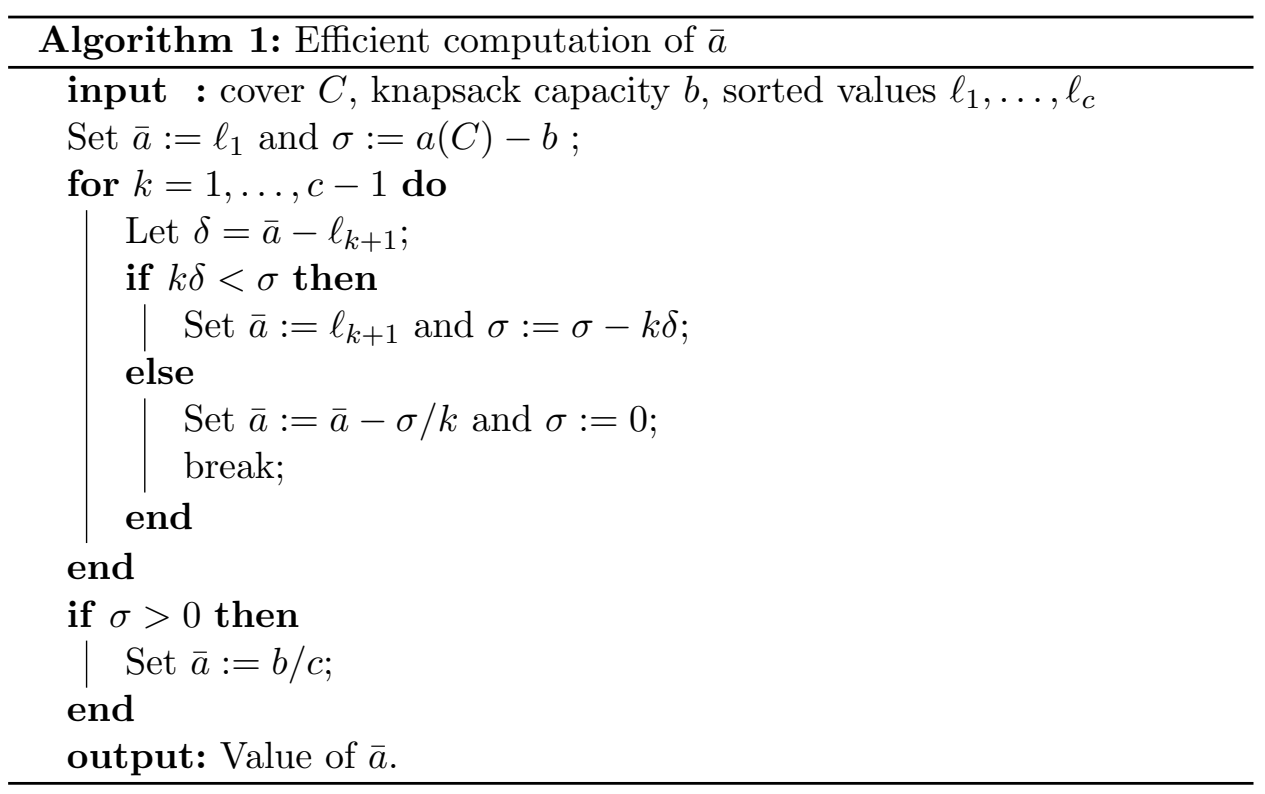

In the remainder of this section, we let $C^{-}=\left\{j \in C: a_{j} \leq \bar{a}\right\}$ and $C^{+}=C \backslash C^{-}$. Note that $C^{-}$can be empty, but $C^{+}$cannot be (since $\left.\bar{a}<a_{\max }\right)$.

\subsection{The improved procedure}

The improved version of Balas' procedure is described in the following theorem.

Theorem 1 For all $j \in C$, let $a_{j}^{-}=\min \left\{a_{j}, \bar{a}\right\}$. For $r=1, \ldots, c$, let $S^{-}(r)$ be the sum of the $r$ largest $a_{j}^{-}$values. (Note that $S^{-}(c)=b$.) Also 
let $S^{-}(0)=0$. Finally, given any $k \in N \backslash C^{-}$, let $\gamma_{j}$ be the largest integer such that $S^{-}\left(\gamma_{j}\right)<a_{k} \leq S^{-}\left(\gamma_{j}+1\right)$. Then the inequality

$$
x\left(C^{-}\right)+\sum_{j \in N \backslash C^{-}} \gamma_{j} x_{j} \leq c-1
$$

is valid for the knapsack polytope, and it is at least as strong as (3).

Proof. First, we expand the definition of "knapsack polytope", by permitting $b$ and/or some of the $a_{j}$ to take fractional values. One can check that Balas up-lifting procedure is valid even in this more general setting. Without loss of generality, we assume that $C \backslash C^{-}=\left\{1, \ldots, c^{\prime}\right\}$ for some $1 \leq c^{\prime} \leq c$. We then define the following "augmented" knapsack polytope:

$$
K^{+}=\operatorname{conv}\left\{x \in\{0,1\}^{n+c^{\prime}}: \sum_{j=1}^{n} a_{j} x_{j}+(\bar{a}+\epsilon) \sum_{j=n+1}^{n+c^{\prime}} x_{j} \leq b\right\},
$$

where $\epsilon$ is some small positive rational number. By construction, the set

$$
\tilde{C}=C^{-} \cup\left\{n+1, \ldots, n+c^{\prime}\right\}
$$

is a cover for $K^{+}$, and it has the same cardinality as $C$. If $\epsilon$ is sufficiently small, then applying Balas' up-lifting procedure to the CI associated with $\tilde{C}$ yields the following LCI for $K^{+}$:

$$
x(\tilde{C})+\sum_{j \in N \backslash C^{-}} \gamma_{j} x_{j} \leq c-1 .
$$

Now, the original knapsack polytope is the face of $K^{+}$obtained by setting $x_{j}$ to zero for $j=n+1, \ldots, n+c^{\prime}$. Thus, the inequality (6) is valid for the original polytope. Finally, note that, by construction, $S^{-}(r)<S(r)$ for all $r$. Thus, $\gamma_{j} \geq \lambda_{j}$ for all $j \in N \backslash C$. Moreover, $\gamma_{j} \geq 1$ for all $j \in C \backslash C^{-}$. Thus, (6) is at least as strong as (3).

As mentioned in the introduction, a peculiarity of the above proof is that it relies on a consideration of "dummy variables" (namely, $x_{n+1}, \ldots, x_{n+c^{\prime}}$ ), which do not actually exist in the original problem.

We now illustrate Theorem 1 on the same example that we considered in Subsection 2.2,

Example 1 (cont.) We have $\bar{a}=4$ and $C=C^{+}$. This means that $S^{-}(k)=4 k$ for $k=0, \ldots, 4$. Since $a_{1}=15>S^{-}(3)$, we have $\gamma_{1}=3$. Since $a_{2}=13>S^{-}(3)$, we have $\gamma_{2}=3$. Since $a_{3}=9>S^{-}(2)$, we have $\gamma_{3}=2$. Finally, since $a_{j}=8<S^{-}(2)$ for $j \in\{4,5,6\}$, we have $\gamma_{j}=1$ for $j \in\{4,5,6\}$. Thus, the resulting (simple) LCI is

$$
3\left(x_{1}+x_{2}\right)+2 x_{3}+x_{4}+\cdots+x_{10} \leq 3 .
$$


This dominates the LCI (5).

Now, observe that, if $\lambda_{1}, \ldots, \lambda_{c}$ have already been computed, then one can compute $S^{-}(0), \ldots, S^{-}(c)$ in $O(c)$ time. Moreover, for each $j \in N \backslash C^{-}$, one can compute $\gamma_{j}$ in $O(\log c)$ time, by binary search. Thus, our improved lifting procedure can be performed in $O(n \log c)$ time.

\subsection{Unusual properties of the new procedure}

As stated in the introduction, our new lifting procedure has some unusual properties. The first is that, if we start with covers that are not minimal, we can obtain LCIs that are not simple. This is illustrated in the following example.

Example 2. Let $n=5, a=(5,5,2,2,2)$ and $b=10$. The cover $C=$ $\{1,2,3,4,5\}$ is not minimal, but we can still apply our procedure. We have $\bar{a}=b / c=2$, so that $S^{-}(r)=2 r$ for $r=0, \ldots, 5$. Now, since $C^{+}=\{1,2\}$, we may be able to increase the coefficients of $x_{1}$ and $x_{2}$. Indeed, since $a_{1}=a_{2}=5>S^{-}(2)$, we have $\gamma_{1}=\gamma_{2}=2$. The resulting valid inequality is $2 x_{1}+2 x_{2}+x_{3}+x_{4}+x_{5} \leq 4$. This is not a simple LCI, since only three variables have a left-hand side coefficient equal to 1 . It is however an LCI, as one can see by setting $C=\{2,3,4,5\}, D=\{2\}, \alpha_{1}=2$ and $\beta_{2}=2$ in (2). It also defines a facet of the associated knapsack polytope.

In general, our procedure yields a simple LCI if and only if at least $|C|$ variables receive a left-hand side coefficient of 1 . One can check that this is equivalent to requiring

$$
\left|\left\{j \in N \backslash C^{-}: \bar{a}<a_{j} \leq S^{-}(2)\right\}\right| \geq\left|C \backslash C^{-}\right| .
$$

In particular, we have the following result.

Lemma 1 If the original cover $C$ is minimal, then our procedure yields a simple $L C I$.

Proof. Let $\sigma=a(C)-b$. Since $C$ is minimal, we have $\sigma \leq \ell_{c} \leq \ell_{2}$. Thus,

$$
a_{\max }=\ell_{1} \leq \ell_{1}+\left(\ell_{2}-\sigma\right)=\left(\ell_{1}+\ell_{2}\right)-\sigma \leq \ell_{1}^{-}+\ell_{2}^{-}=S^{-}(2) .
$$

This implies that $\gamma_{k}=1$ for all $k \in C \backslash C^{-}$.

An even more unusual property of the new procedure is that, if we start with covers that are not minimal, we can obtain inequalities that are not LCIs at all (in the traditional sense).

Example 3. Let $n=5, a=(10,7,7,4,4)$ and $b=16$. The cover $C=$ 


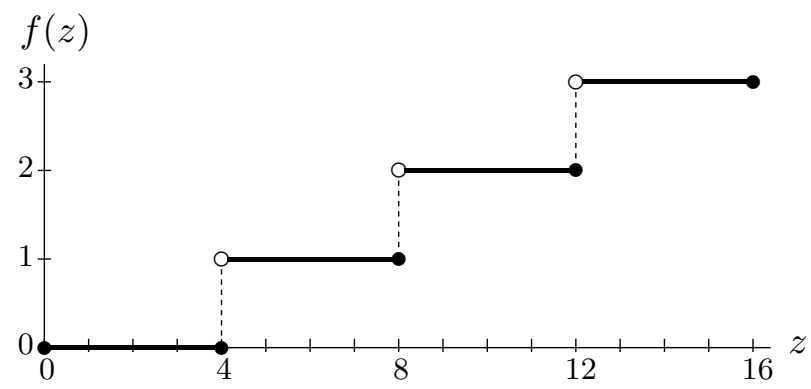

Figure 1: The lifting function $f(z)$ for Example 1 .

$\{1, \ldots, 5\}$ is not minimal, but we apply our procedure. We have $\bar{a}=b / c=$ $3 \frac{1}{5}$, so that $S^{-}(0), \ldots, S^{-}(5)$ are $0,3 \frac{1}{5}, 6 \frac{2}{5}, 9 \frac{3}{5}, 12 \frac{4}{5}$ and 16 . Now, since $\mathrm{C}^{+}=C$, we may be able to increase the coefficients for some of the variables in the cover. One can check that we get $\gamma_{1}=3, \gamma_{2}=\gamma_{3}=2$ and $\gamma_{4}=\gamma_{5}=1$. The resulting valid inequality is

$$
3 x_{1}+2 x_{2}+2 x_{3}+x_{4}+x_{5} \leq 4
$$

One can check (by brute-force enumeration of all possible lifting orders) that this inequality cannot be obtained from a CI by standard lifting methods, sequential or otherwise. One can also check (either by hand or with the help of a software package such as PORTA [6]) that it defines a facet of the associated knapsack polytope.

\section{Additional Improvement Via Superadditivity}

A further improvement in the lifting procedure can be achieved from a consideration of superadditive lifting functions. The lifting function associated with Theorem 1 is:

$$
f(z)= \begin{cases}0 & \text { if } z=0 \\ h & \text { if } S^{-}(h)<z \leq S^{-}(h+1) \text { for some } h=0, \ldots, c-1\end{cases}
$$

where the domain of $z$ is understood to be $[0, b]$. (Figure 1 shows the function $f(z)$ for Example 1.)

Our goal is to construct an even stronger lifting function. We will need the following three results.

Lemma 2 The function $f$ is superadditive on its domain.

Proof. Let $z, z^{\prime} \in[0, b]$ be such that $z+z^{\prime} \leq b$. Suppose that $f(z)=\gamma$ and $f\left(z^{\prime}\right)=\gamma^{\prime}$. Then, by definition, we have $z>S^{-}(\gamma)$ and $z^{\prime}>S^{-}\left(\gamma^{\prime}\right)$. 
Let $\ell_{1}^{-}, \ldots, \ell_{c}^{-}$be the $a_{j}^{-}$values sorted in non-increasing order. We have $z>\sum_{j=1}^{\gamma} \ell_{j}^{-}$and $z^{\prime}>\sum_{j=1}^{\gamma^{\prime}} \ell_{j}^{-}$. We then have:

$$
z+z^{\prime}>\sum_{j=1}^{\gamma} \ell_{j}^{-}+\sum_{j=1}^{\gamma^{\prime}} \ell_{j}^{-} \geq \sum_{j=1}^{\gamma+\gamma^{\prime}} \ell_{j}^{-}
$$

where the second inequality follows from the fact that the $\ell_{j}^{-}$are sorted in non-increasing order. Thus, $f\left(z+z^{\prime}\right) \geq \gamma+\gamma^{\prime}=f(z)+f\left(z^{\prime}\right)$.

Lemma 3 The upper bound of Balas and Zemel [3] remains valid even when the cover $C$ is not minimal. That is, for any $k \in N \backslash C$, the lifting coefficient of $x_{k}$ cannot exceed $\mu_{k}$, where $\mu_{k}$ is the unique integer such that (4) holds.

Proof. The lifting coefficient achieves its maximum possible value when $x_{k}$ is lifted first. Note that, if we set $x_{k}$ to 1 , the remaining capacity in the knapsack is $b-a_{k}$. Then, the maximum value that $x(C)$ can take is equal to the largest integer $s$ such that $\sum_{j=c-s+1}^{c} \ell_{j} \leq b-a_{k}$. But $\sum_{j=c-s+1}^{c} \ell_{j}=$ $a(C)-S(c-s)$. Since the right-hand side of the CI is $c-1$, the lifting coefficient cannot exceed $(c-1)-s$, which is nothing but $\mu_{k}$.

Lemma 4 The function $f$ reaches the Balas-Zemel bound when $z \geq b-$ $a\left(C^{-}\right)$.

Proof. Note that $\gamma_{j}$ is the unique integer such that $S^{-}\left(\gamma_{j}\right)<a_{j} \leq$ $S^{-}\left(\gamma_{j}+1\right)$, and $\mu_{j}$ is the unique integer such that $S\left(\mu_{j}\right)+b-a(C)<a_{j} \leq$ $S\left(\mu_{j}\right)+b-a(C)$. But $S^{-}(C)=b=S(C)+b-a(C)$, which implies that $S^{-}(k)=S(k)+b-a(C)$ for $k \geq\left|C^{+}\right|$. So $\gamma_{j}=\mu_{j}$ for $z \geq b-a\left(C^{-}\right)$.

Lemma 4 leaves open the possibility that the value of $f(z)$ could be increased for some values of $z$ smaller than $b-a\left(C^{-}\right)$. This is indeed the case.

Theorem 2 The following function is a superadditive valid lifting function:

$$
g(z)= \begin{cases}f(z)+\frac{1}{2} & \text { if } z=h \bar{a} \text { for some integer } h \in\left[1,\left|C^{+}\right|-1\right] \\ f(z) & \text { otherwise. }\end{cases}
$$

(See Figure 2 for an illustration.)

Proof. From the results in Wolsey [17], we need to prove that (a) $g$ is superadditive and (b) $g(z)$ never exceeds the Balas-Zemel bound.

First, we prove superadditivity. Since $f$ is superadditive, we need to prove only that $g(z)+g\left(z^{\prime}\right) \leq g\left(z+z^{\prime}\right)$ when $g(z)>f(z)$, i.e., when $z=h \bar{a}$ for some integer $h \in\left[1,\left|C^{+}\right|-1\right]$. We have $z=\sum_{j=1}^{h} \ell_{j}^{-}$, where $\ell_{j}^{-}$is defined as in the proof of Lemma 2. We also have $g(z)=h-\frac{1}{2}$. We consider two cases. 


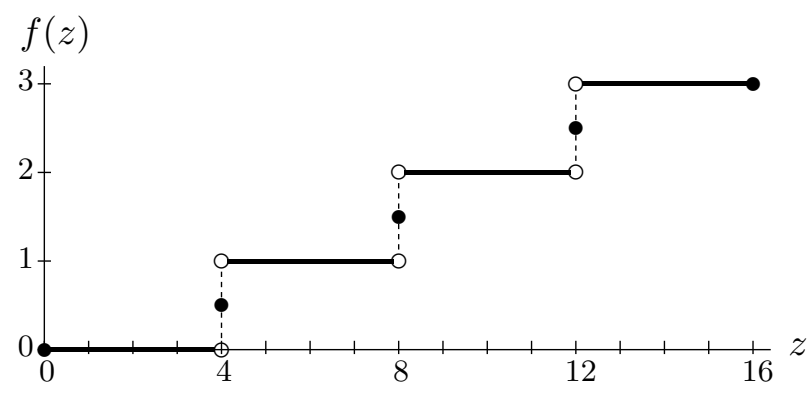

Figure 2: The improved lifting function $g(z)$ for Example 1.

1. $g\left(z^{\prime}\right)=f\left(z^{\prime}\right)=\gamma^{\prime}$. In this case, we have $z^{\prime}>\sum_{j=1}^{\gamma^{\prime}} \ell_{j}^{-}$. This implies

$$
z+z^{\prime}>\sum_{j=1}^{h} \ell_{j}^{-}+\sum_{j=1}^{\gamma^{\prime}} \ell_{j}^{-} \geq \sum_{j=1}^{h+\gamma^{\prime}} \ell_{j}^{-},
$$

which in turn implies $g\left(z+z^{\prime}\right) \geq h+\gamma^{\prime}>h-\frac{1}{2}+\gamma^{\prime}=g(z)+g\left(z^{\prime}\right)$.

2. $g\left(z^{\prime}\right)=f\left(z^{\prime}\right)+\frac{1}{2}$. In this case, $z=h^{\prime} \bar{a}$ for some integer $h^{\prime} \in\left[1,\left|C^{+}\right|-\right.$ 1]. This implies

$$
z+z^{\prime}=\sum_{j=1}^{h} \ell_{j}^{-}+\sum_{j=1}^{h^{\prime}} \ell_{j}^{-} \geq \sum_{j=1}^{h+h^{\prime}} \ell_{j}^{-},
$$

which in turn implies $g\left(z+z^{\prime}\right) \geq h+h^{\prime}-1=\left(h-\frac{1}{2}\right)+\left(h^{\prime}-\frac{1}{2}\right)=$ $g(z)+g\left(z^{\prime}\right)$.

Now we show that $g(z)$ never exceeds the Balas-Zemel bound. Since $f$ is a valid lifting function, it follows that $f(z)$ never exceeds the bound. The only time that $g(z)>f(z)$ is when $z=h \bar{a}$ for some integer $h \in\left[1,\left|C^{+}\right|-1\right]$. In this case, we have

$$
b-z=b-h \bar{a}=\sum_{j=h+1}^{c} \ell_{j}^{-}<\sum_{j=h+1}^{c} \ell_{j}=a(C)-S(h) .
$$

Together with (4), this means that the Balas-Zemel upper bound is at least $h$. This exceeds $g(h \bar{a})=h-\frac{1}{2}$.

It turns out that using $g(z)$ in place of $f(z)$ can lead to stronger LCIs, even when the $a_{j}$ are integers and the cover is minimal.

Example 1 (cont.) We have $\bar{a}=4$ and $\left|C^{+}\right|=4$. Setting $h=2$ in Theorem 2, we obtain $g(2 \bar{a})=g(8)=3 / 2$. This yields the stronger LCI

$$
3\left(x_{1}+x_{2}\right)+2 x_{3}+\frac{3}{2}\left(x_{4}+x_{5}+x_{7}\right)+x_{7}+\cdots+x_{10} \leq 3 .
$$


This LCI can be shown to be facet-defining.

We also have the following result:

Proposition 1 The function $g(z)$ is non-dominated (that is, there does not exist a superadditive valid lifting function that is stronger than $g(z))$.

Proof. Lemma 4 shows that $g(z)$ cannot be increased when $z \geq b-$ $a\left(C^{-}\right)=\left|C^{+}\right| \bar{a}$. One can check that, for any pair $\left(z, z^{\prime}\right)$ such that $z+z^{\prime}=$ $\left|C^{+}\right| \bar{a}$, we have $g(z)+g\left(z^{\prime}\right)=g\left(\left|C^{+}\right| \bar{a}\right)=\left|C^{+}\right|-1$. Thus, $g(z)$ cannot be increased when $z<\left|C^{+}\right| \bar{a}$ either.

Note that $g(z)$ is half-integral, and integer-valued almost everywhere. We found it surprising that a non-dominated lifting function with these properties exists. (Indeed, the lifting function presented in [10] is integervalued only on certain intervals.)

We know of some other superadditive valid lifting functions that dominate $f(z)$. The one that we find most interesting is presented in the following proposition.

Proposition 2 If $a_{j} \neq \bar{a}$ for all $j \in C$, the following function is a nondominated superadditive valid lifting function:

$$
g^{\prime}(z)= \begin{cases}f(z)+1 & \text { if } z=h \bar{a} \text { for some integer } h \in\left(\left|C^{+}\right| / 2,\left|C^{+}\right|-1\right] \\ f(z)+\frac{1}{2} & \text { if }\left|C^{+}\right| \text {is even and } z=\left|C^{+}\right| \bar{a} / 2 \\ f(z) & \text { otherwise. }\end{cases}
$$

Proof. The proof is similar to that of Theorem 2 and Proposition 1 . The key difference occurs when $g^{\prime}(z)=f(z)+1$ and $g^{\prime}\left(z^{\prime}\right)>f\left(z^{\prime}\right)$. In this case, let $z=h \bar{a}$ and $z^{\prime}=h^{\prime} \bar{a}$. Since $h$ exceeds $\left|C^{+}\right| / 2$ and $h^{\prime}$ is at least $\left|C^{+}\right| / 2$, we must have $z+z^{\prime}>\left|C^{+}\right| \bar{a}$. Moreover, if $a_{j} \neq \bar{a}$ for all $j \in C$, we have $S^{-}(k)<k \bar{a}$ for all $k>\left|C^{+}\right|$. This implies that $g^{\prime}\left(z+z^{\prime}\right) \geq h+h^{\prime}=$ $g^{\prime}(z)+g^{\prime}\left(z^{\prime}\right)$.

This lifting function is integer-valued at all points when $\left|C^{+}\right|$is even.

\section{Acknowledgements}

The second author gratefully acknowledges financial support from the EPSRC through the STOR-i Centre for Doctoral Training under grant EP/L015692/1.

\section{References}

[1] A. Atamtürk (2005) Cover and pack inequalities for (mixed) integer programming. Ann. Oper. Res., 139, 21-38. 
[2] E. Balas (1975) Facets of the knapsack polytope. Math. Program., 8, 146-164.

[3] E. Balas \& E. Zemel (1978) Facets of the knapsack polytope from minimal covers. SIAM J. Appl. Math., 34, 119-148.

[4] M. Conforti, G. Cornuéjols \& G. Zambelli (2015) Integer Programming. Graduate Texts in Mathematics, vol. 271. Springer.

[5] D.-S. Chen, R.G. Batson \& Y. Dang (2011) Applied Integer Programming. Hoboken, NJ: Wiley.

[6] T. Christof \& A. Loebl, PORTA (polyhedron representation transformation algorithm). Software package, available for download at http://www.iwr. uni-heidelberg.de/groups/comopt/software

[7] H. Crowder, E. Johnson \& M. Padberg (1983) Solving large-scale 0-1 linear programming programs. Oper. Res., 31, 803-834.

[8] F. Glover (1973) Unit-coefficient inequalities for zero-one programming. Management Science Report 73-7, University of Colorado, July 1973.

[9] Z. Gu, G.L. Nemhauser \& M.W.P. Savelsbergh (1998) Lifted cover inequalities for 0-1 integer programs: computation. INFORMS J. Comput., 10, 427-437.

[10] Z. Gu, G.L. Nemhauser \& M.W.P. Savelsbergh (2000) Sequenceindependent lifting in mixed integer programming. J. Comb. Optim., $4,109-129$.

[11] D. Hartvigsen \& E. Zemel (1992) The complexity of lifted inequalities for the knapsack problem. Discr. Appl. Math., 39, 113-123.

[12] K.L. Hoffman \& M.W. Padberg (1991) Improving LP-representations of zero-one linear programs for branch-and-cut. ORSA J. Comput., 3, $121-134$.

[13] K. Kaparis \& A.N. Letchford (2010) Separation algorithms for 0-1 knapsack polytopes. Math. Program., 124, 69-91.

[14] K. Kaparis \& A.N. Letchford (2011) Cover inequalities. In J.J. Cochran et al. (eds.) Encyclopedia of Operations Research and Management Science. New York: Wiley.

[15] M.W. Padberg (1975) A note on zero-one programming. Oper. Res., $23,833-837$.

[16] L.A. Wolsey (1975) Faces for a linear inequality in 0-1 variables. Math. Program., 8, 165-178. 
[17] L. Wolsey (1977) Valid inequalities and superadditivity for 0-1 integer programs. Math. Oper. Res., 2, 66-77.

[18] E. Zemel (1989) Easily computable facets of the knapsack polytope. Math. Oper. Res., 14, 760-765. 